Randomized Trial

\title{
Automatic Adaptation of Neurostimulation Therapy in Response to Changes in Patient Position: Results of the Posture Responsive Spinal Cord Stimulation (PRS) Research Study
}

Cristy M. Schade, MD, PhD, PE ${ }^{1}$, David Schultz, MD², Nancy Tamayo, DC', Sudha lyer, PhD², and Eric Panken, $\mathrm{MS}^{3}$

From: ${ }^{1}$ Center for Pain Control, Garland, TX; ${ }^{2}$ MAPS Applied Research Center, Edina, MN; ${ }^{3}$ Medtronic Neuromodulation, Minneapolis, MN

Dr. Schade is with the Center for Pain Control, Garland, TX. Dr. Schultz is medical director of MAPS Applied Research Center, Edina, MN. Dr. Tamayo is from the Center for Pain Control,

Garland, TX. Dr. Iyer is a clinical research manager with Medtronic Neuromodulation., Minneapolis, MN. Mr. Panken is senior principal scientist with Medtronic Neuromodulation, Minneapolis, MN

Address correspondence: Cristy M. Schade, MD, PhD, PE Center for Pain Control 2692 W. Walnut Street, Suite 105 Garland, TX 75042

E-mail: CMSchade@cpctx.com

Disclaimer: For full disclosure see page 416

Manuscript received: 05/31/2011 Accepted for publication: 07/22/2011

Free full manuscript: www.painphysicianjournal.com
Background: Variation in the intensity of neurostimulation with body position is a practical problem for many patients implanted with a spinal cord stimulation system because positional changes may result in overstimulation or understimulation. These posture-related changes in patients' perception of paresthesia can affect therapeutic outcomes of spinal cord stimulation therapy. An accelerometer-based algorithm that automatically adjusts spinal cord stimulation based on sensed body position or activity represents a potential solution to the problem of position-mediated variations in paresthesia perception.

Objective: The objective of this study was to compare patient satisfaction ratings for manual versus automatic adjustment of spinal cord stimulation amplitude in response to positional changes

Study Design: Prospective, multicenter, open-label, randomized trial

Setting: 2 pain centers in the US.

Method: Twenty patients at 2 centers in the U.S. who had been implanted with a spinal cord stimulation system for low back and/or leg pain were enrolled in the study. During a 3-day run-in phase, patient position and activity changes were monitored with an ambulatory data recorder and with a research patient programmer which recorded all stimulation parameter changes. Patients who made $\geq 2$ amplitude adjustments per 24hour period were invited to participate in an in-clinic phase. During the in-clinic phase, patients' preferred stimulation amplitude and therapy impedance measured at the preferred stimulation amplitude were determined as they performed a series of 8 physical tasks. Satisfaction ratings were determined during position transitions between the physical tasks using both manual and automatic adjustments.

Results: Among the 15 patients who completed the in-clinic test protocol, overall satisfaction ratings were significantly higher for automatic adjustment of stimulation amplitudes versus manual adjustments. Patients reported statistically significant improvements with automatic versus manual adjustment for the standing to supine transition and for supine to standing transition. Approximately $74 \%$ of participants rated the paresthesia intensity of the automatic adjustment algorithm as "just right" for the physical tasks that were completed.

Limitations: Small study size.

Conclusion: Patients preferred automatic versus manual adjustment of stimulation amplitude in response to changes in paresthesia consequent to positional changes during in-clinic testing.

Key words: spinal cord stimulation, automaticity, paresthesia, neuromodulation, neurostimulation, adaptive stimulation, posture responsive stimulation, accelerometry

Pain Physician 2011; 14:407-417 
S pinal cord stimulation (SCS) is an accepted and increasingly common therapy for the treatment of various types of chronic pain of the trunk and limbs. The most frequent indication is chronic low back and/or leg pain that persists despite corrective back surgery (1-4). Recent systematic reviews (1), and prospective, randomized, controlled studies (5-7) have demonstrated that SCS therapy provides significant long-term pain relief in carefully selected patients. SCS therapy generates paresthesia that reduces the perception of pain. For maximum efficacy, the paresthesia sensation must substantially cover the patient's pain distribution, and the patient must perceive the paresthesia intensity as comfortable. If paresthesia coverage does not provide sufficient overlap with a patient's pain, therapy success will be compromised (8). Similarly, paresthesia intensity should be delivered at a power that is both effective for treatment and comfortable to the patient (9). Paresthesia levels that are too weak will be ineffective, while levels that are too intense might be perceived as painful.

Recent technological advances facilitate tailoring neurostimulation therapy to meet individual patient needs. Sophisticated programming options can adapt neurostimulation therapy to changing pain patterns (10). With the newest neurostimulators, the electrical field can be shaped using combinations of anodes and cathodes on multiple leads. Rechargeable devices permit higher stimulation parameters without compromising device longevity. Similarly, patient programmers now make it possible for patients themselves to adjust their therapy (within clinician-prescribed limits) to a degree previously not possible. Because patients typically experience changes in their perception of paresthesia over time (11), these programming options can improve the likelihood of long-term effective pain management.

Several studies have documented changes in the location and intensity of paresthesia due to position changes $(9,12,13)$. These changes in paresthesia intensity might be due to changes in the distance between the lead in the epidural space and the dorsal column fibers (13-15). Paresthesia perception during movement from the standing to supine position is particularly affected for many patients. For example, a patient might find that the paresthesia intensity that provides the best pain coverage and comfort for standing might be too intense in the supine position. Conversely, the paresthesia intensity in the supine position might provide inadequate pain relief when the patient is standing. While patients can use their patient programmer to adjust stimulation levels to improve comfort, making adjustments multiple times per day can affect quality of life. Furthermore, even though technology improvements have made patient programmers more powerful and easier to use, not all patients are equally adept at using them. Thus, some patients endure ongoing suboptimal pain relief because of insufficient use of their patient programmers. This paper reports the results of the Posture Responsive Spinal Cord Stimulation (PRS) study, a prospective, multicenter, open-label, randomized study to examine the feasibility and utility of an accelerometer-based algorithm that automatically adjusts spinal cord stimulation based on position changes or activity. Automatic adjustment of stimulation amplitude might be referred to as adaptive stimulation also in this paper.

\section{Methods}

\section{Institutional Review Board and Patient Informed Consent}

This study enrolled 20 patients at 2 centers in the United States between August 2008 and January 2009. The Institutional Review Board at each site approved the protocol. All patients signed a study-specific informed consent form prior to study enrollment.

\section{Patient Selection}

Eligible patients were ambulatory and at least 18 years of age. They suffered from chronic low back pain and/or leg pain due to neuropathic causes and had been implanted with a neurostimulation system (Restore $®$ or Restore Advanced $®$, Medtronic Inc., Minneapolis, MN) with percutaneous leads for at least 3 months. Patients demonstrated stable pain control with their neurostimulation system (defined as satisfaction with current settings for at least one post-implant programming session) and used their patient programmer to adjust stimulation amplitude at least 2 times per 24hour period for position changes or activity during a 3-day run-in phase. Patients were excluded if they were pregnant, morbidly obese (body mass index $\geq 40$ ), had pain-related surgery in the 12 weeks prior to enrollment, or intended to undergo surgery during the study period.

\section{Study Design}

The study design is depicted in Fig. 1. The study encompassed both out of clinic and in-clinic phases. During an initial run-in phase, baseline demographic data were collected on consenting study participants and the 


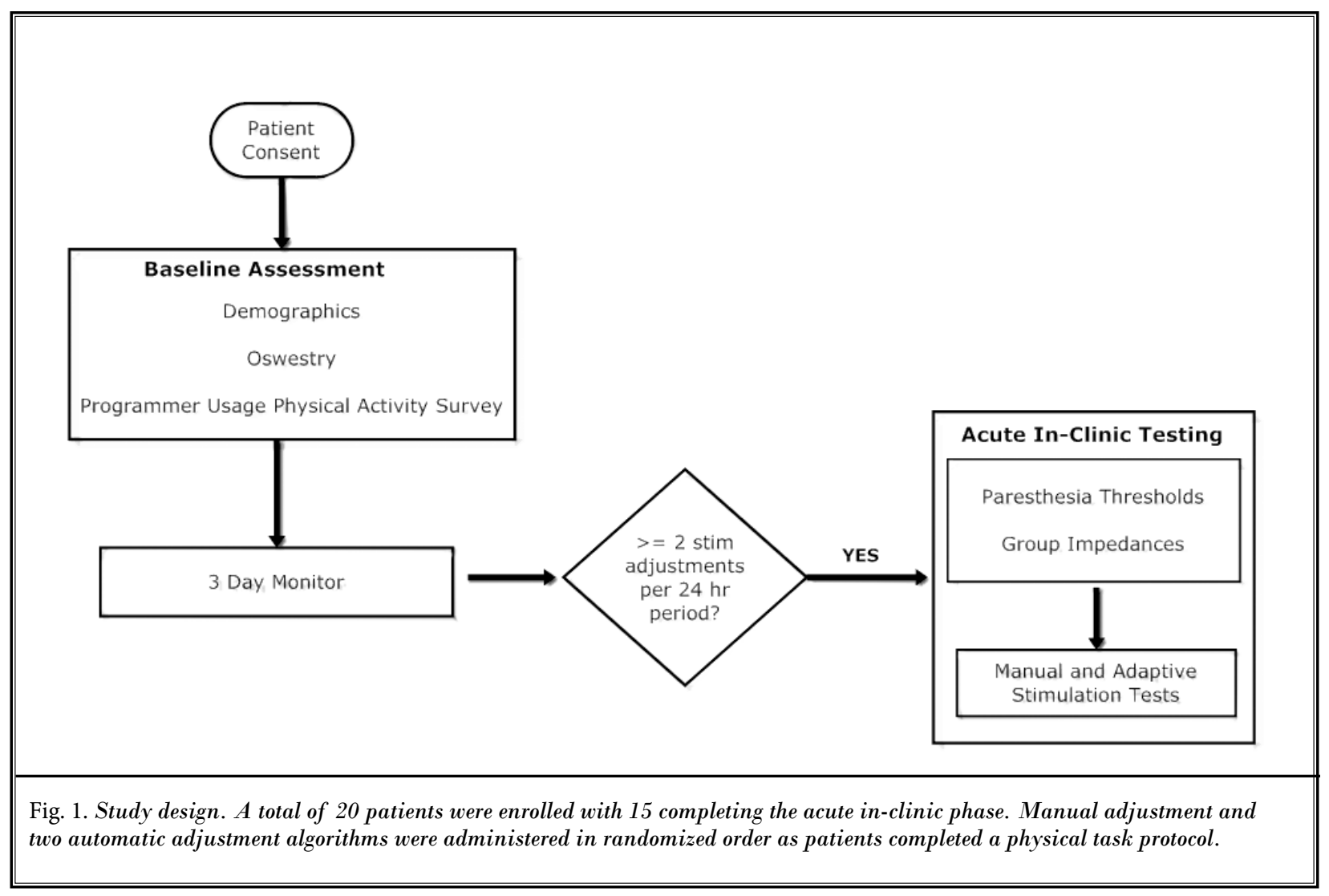

Oswestry Disability Index (ODI) was administered to assess patient function. Participants were then fitted with an ambulatory data recorder (Micro ADR, Medtronic, Inc., Minneapolis, MN), which was calibrated and used to monitor participants' position changes and activity for 3 days in an outpatient setting. Patient programmer usage was also recorded during this time period.

\section{3-Day Monitoring Phase}

The purpose of the out-of-clinic 3-day monitoring phase was to ensure that participants in the acute inclinic phase of the study used their patient programmer to change the stimulation amplitude in response to changes in body position or activity. Participants were also provided with electronic diaries to record activities of daily living and research patient programmers that logged every stimulation parameter adjustment. Based on analysis of the 3-day monitoring data, patients who made at least 2 stimulation amplitude adjustments per 24-hour period due to position changes or activity were invited to participate in the in-clinic phase of the PRS study.

\section{In-Clinic Phase}

The purpose of the in-clinic phase was to test automatic adjustments and to compare satisfaction ratings between automatic and manual adjustments. During this phase, the participant's implantable neurostimulator was interrogated to record baseline stimulation parameters. Paresthesia thresholds (perceptual and unacceptably strong) were assessed in the following positions: standing, sitting, lying supine, lying on right side, lying prone, lying on left side, reclining at $45^{\circ}$, and walking on a treadmill. Following these assessments the PRS Study instrumentation was fitted and the sensor module calibrated to the participant's body by taking accelerometer measurements in the upright, supine, prone, on left, and on right lying positions. Patient-preferred amplitudes in the supine, prone, on left, on right, upright, and upright mobile were also determined. Thus, during the adaptive stimulation tests the implantable neurostimulator automatically adjusted the stimulation amplitude based on the sensed body position and the patient preferred stimulation amplitudes. 


\section{PRS Study Instrumentation}

The in-clinic study instrumentation used to execute adaptive stimulation is shown in Fig. 2. The Micro ADRII, a bandage-mounted sensor module containing a single ADXL-330 triaxial accelerometer from Analog Devices (Norwood MA), measured body acceleration. The sensor modules were attached to the body of each study patient with hypo-allergenic foam tape specifically designed for medical applications. The sensor module transmitted data wirelessly to the ADRII device via telemetry using the $433 \mathrm{MHz}$ ISM (Industrial, Scientific, and Medical) band. The ADRII device interfaced to the PRS study programmer via a USB port providing the study programmer with real-time access to the sensor signals. The Micro-ADRII and ADR II devices were battery-powered and nontherapeutic.

The PRS study programmer consisted of a batterypowered personal computer and custom telemetry module. The PRS study programmer software application executed adaptive stimulation via the telemetry module (Fig. 3).

Participants used a study-specific patient programmer to adjust stimulation amplitudes during manual adjustment protocol tasks. This study patient programmer was identical to the commercially-available programmer used with the Restore and Restore Advanced neurostim-

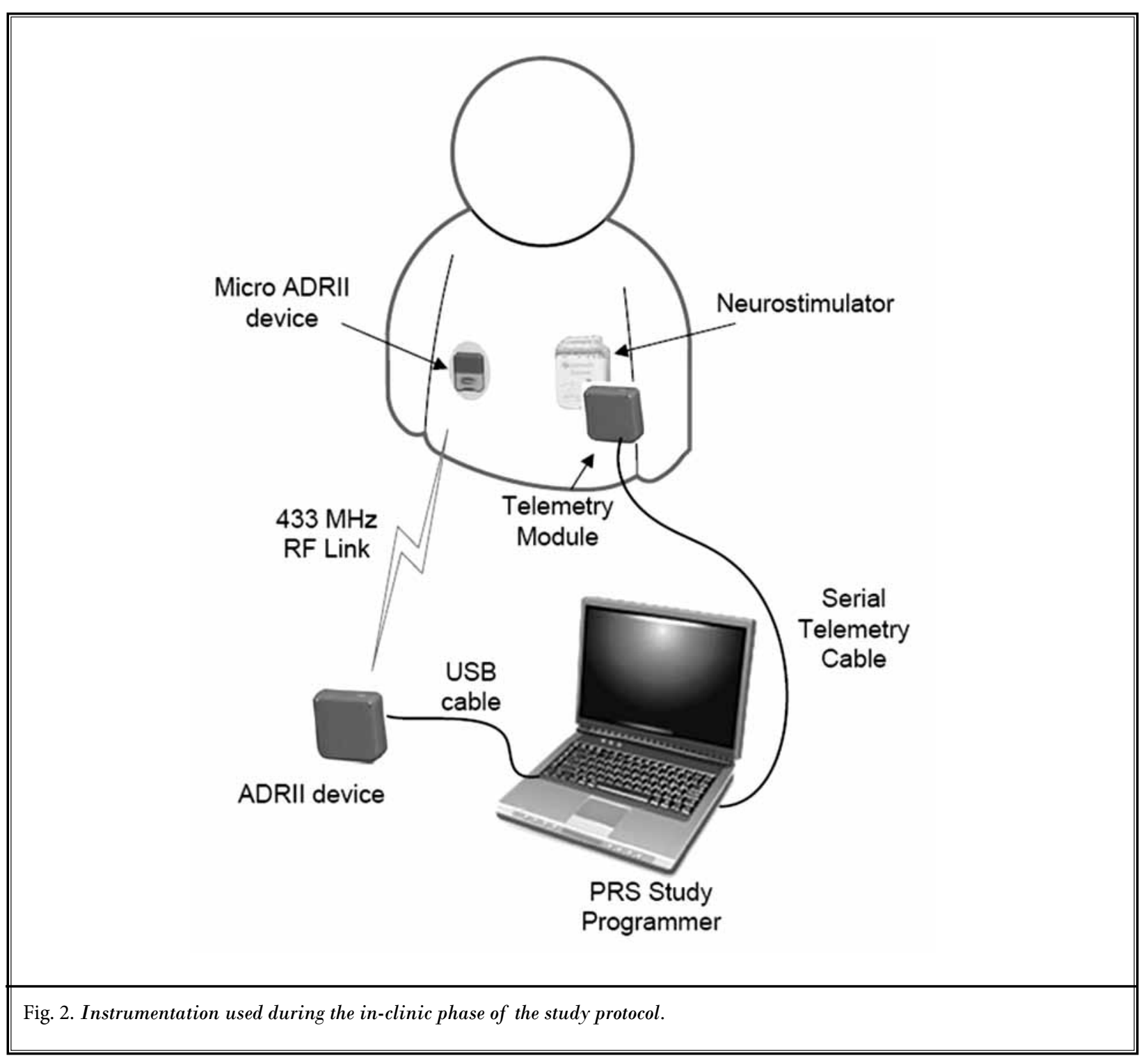




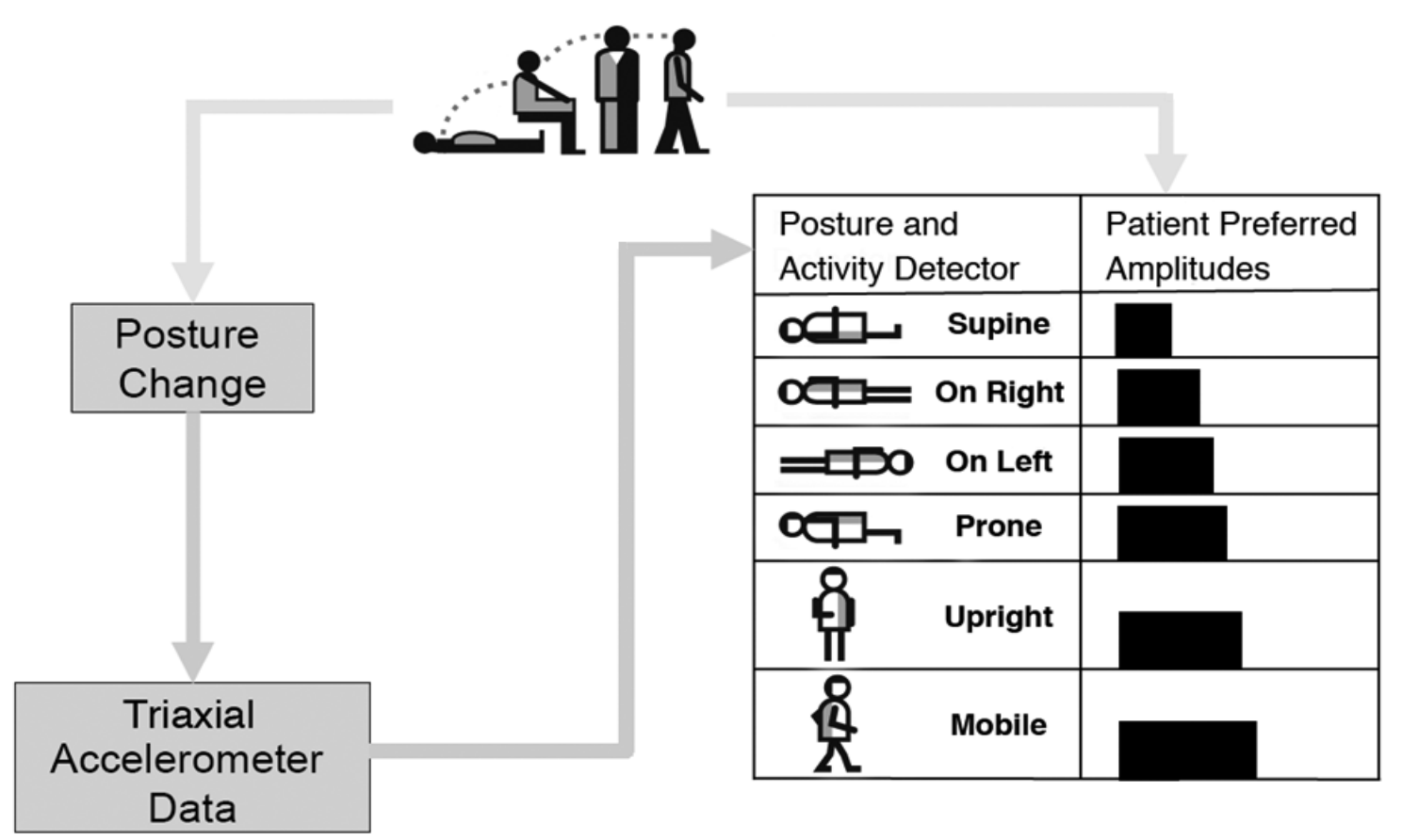

Fig. 3. The adaptive stimulation algorithms detected when the patient was supine, prone, lying on left side, lying on right side, upright, and active (mobile) and automatically adjusted the stimulation intensity based on the sensed body position and activity and patient-preferred amplitudes.

ulators in all respects except for the firmware which had been modified to record all programming changes made by the patient. The commercially-available Model 8840 $\mathrm{N}$ 'Vision clinician programmer (Medtronic Inc., Minneapolis, MN) was used to interrogate and program the participant's implantable neurostimulator.

\section{In-Clinic Physical Task Protocol}

The patient-preferred amplitudes and associated therapy impedances were determined for the series of 8 physical tasks described previously. Patients repeated the physical tasks in the protocol 3 times: once with manual adjustment using the patient programmer and once with each of the two adaptive stimulation algorithms under test. The algorithms differed in how the transition between lying and upright was handled. One algorithm implemented an adjustable transition zone with hysteresis between the lying and upright positions, while the other continuously adjusted stimulation amplitude as a function of the recline angle between lying and upright positions.
Patient satisfaction ratings were collected during the different position transitions for manual adjustment and for adaptive stimulation. Paresthesia satisfaction ratings were recorded using a standard 7-point numeric Likert scale with 1 being very dissatisfied and 7 being very satisfied. Paresthesia intensity was assessed as being low, just right, and high during each of the position transitions that occurred during the automatic adjustment protocol. Paresthesia intensity was not rated during the manual protocol, as participants could adjust stimulation to their preferred levels.

\section{Randomization}

Each participant completed the 8 physical tasks repetitively under manual stimulation and under each of the 2 adaptive stimulation algorithms. The order of the physical tasks was randomized for each sequence. During the adaptive stimulation sequence, the algorithm order was also randomized with participants blinded to the type of adaptive stimulation algorithm deployed. 


\section{Statistical Analysis}

Based on a study of postural changes in SCS and sample-size calculations, we determined a sample of 15 to 30 patients would be adequate to meet the feasibility goals of the study (13). Each patient could have up to 4 active programs in a group for SCS therapy. Each active program had its own stimulation voltage. Voltage is reported as a percentage of the voltage in the standing position. Impedance is also reported as percentage of impedance measured in the standing position. Mean voltage and mean impedance were averaged over active SCS programs and reported per patient and position. Overall mean voltage and impedance were calculated as the average mean voltage and mean impedance across patients and reported per position.

All patients who provided a satisfaction rating for manual and automatic adjustments or a paresthesia intensity rating for automatic adjustments during position changes were included in the analysis. A paired $t$ test was used to compare satisfaction ratings of manual and automatic adjustment from the standing to the supine position and from the supine to the standing position. Friedman's test was used to compare manual versus automatic adjustment satisfaction ratings across all positions. $P$ values $\leq 0.05$ were considered statistically significant. Statistical analyses were performed using SAS and Minitab software package(s), (SAS Inc., Cary, NC, Minitab Inc., State College, PA).

\section{Results}

\section{Patients}

A total of 20 patients ( 6 men and 14 women) from 2 centers were enrolled in the study between August 2008 and January 2009. The mean age of enrolled participants was 54.4 years (range 43-66 years). The average duration of SCS therapy was 5.9 years (range 0.3-18.4 years). The primary pain etiology was failed back surgery syndrome $(n=18)$. The mean ODI was 52.3 (range 36-66). Characteristics of the study population were similar to those reported in other studies of spinal cord stimulation $(6,16)$. Of the 20 enrolled patients, one was discontinued from the study prior to the 3-day monitoring period due to eligibility criteria not being met. Of the 19 participants completing the 3-day at-home monitor, one failed the minimum stimulation adjustment criteria and 1 withdrew consent. Seventeen patients were invited to participate in the in-clinic testing; one participant withdrew due to an adverse event unrelated to the study and one participant failed to complete the in-clinic testing due to scheduling conflicts and a failure of the participant to bring their patient programmer to the clinic. Thus, 15 participants provided complete data for the comparisons between manual and automatic adjustments.

\section{Manual Versus Automatic Adjustment}

Among the 15 participants who provided complete data for the comparisons between manual and automatic adjustments, patients preferred automatic over manual adjustment. Two adaptive stimulation algorithms were tested, and both were preferred over manual adjustment, as shown by overall satisfaction rating scores. We report the results for the algorithm that was selected for further evaluation in an implantable neurostimulation system. The overall mean satisfaction rating scores were 5.04 (SD 1.91) for manual and 6.16 (SD 1.03) for automatic adjustment across all positions (Fig. 4). The difference in satisfaction rating between manual and automatic adjustment across all positions was statistically significant $(P<0.001$, Friedman's test).

\section{Standing to Supine and Supine to Standing Transitions}

Maintaining optimal paresthesia coverage during the transitions from the standing to supine and supine to standing positions has posed particular challenges for patients. Thus, these transitions were studied in greatest detail in this study. The satisfaction ratings for these 2 position transitions showed that participants $(n=15)$ experienced statistically significant improvements with the automatic adjustment algorithm when compared to manual adjustments (Fig. 5). The mean satisfaction rating after the standing to supine transition was 4.14 (SD 2.11) for manual adjustment and 6.0 (SD 1.07) for automatic adjustment. The mean improvement in the satisfaction score for automatic adjustment over manual adjustment was 1.86 (SD 1.86) ( $p=0.01$, paired $t$-test). For the supine to standing transition, the mean satisfaction rating was 5.07 (SD 1.98) for manual adjustment and 6.47 (SD 0.64) for automatic adjustment for a mean improvement of 1.40 (SD 2.13) ( $P=0.023$, paired t-test).

\section{Paresthesia Intensity for Automatic Adjustment}

Patients rated the paresthesia intensity of the automatic adjustment algorithm "just right" for $74.1 \%$, too low for $12.8 \%$, and too high for $13.1 \%$ of the physical tasks completed. 


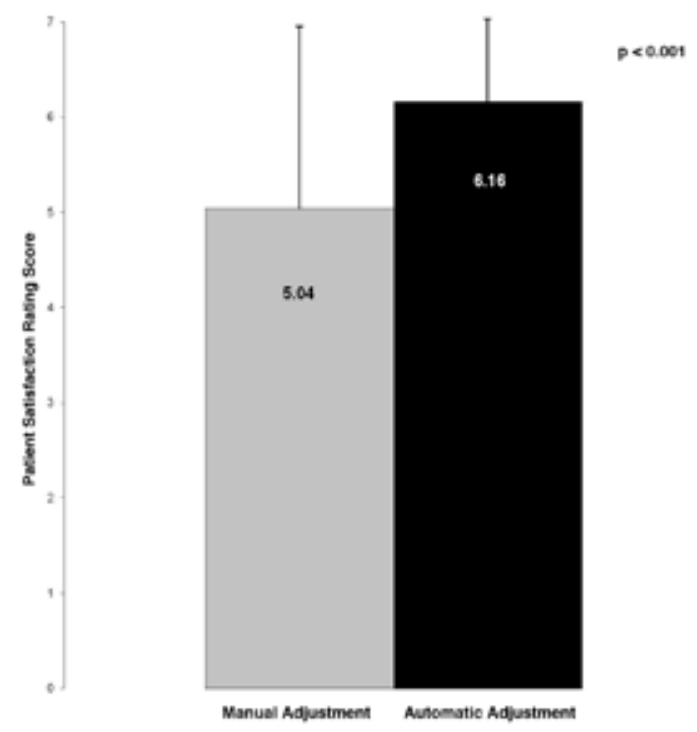

Fig. 4. Overall mean (+1 SD) satisfaction ratings for manual versus automatic adjustment across all positions is shown. The difference in satisfaction rating between manual and automatic adjustment across all positions was statistically significant $(P$ $<0.001$, Friedman's test). Satisfaction ratings are shown on the 7-point Likert rating with 0 being very dissatisfied and 7 being very satisfied.

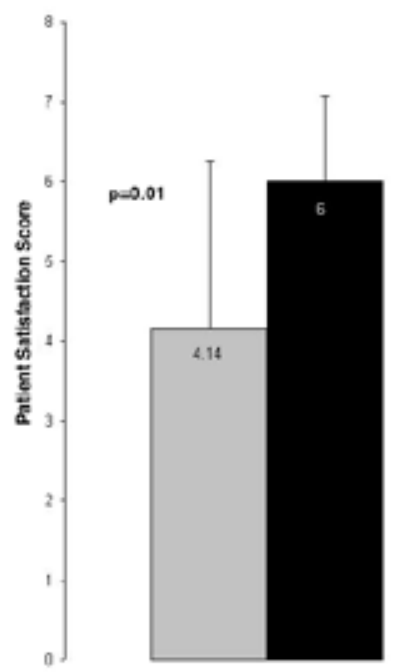

Stued To Sephet

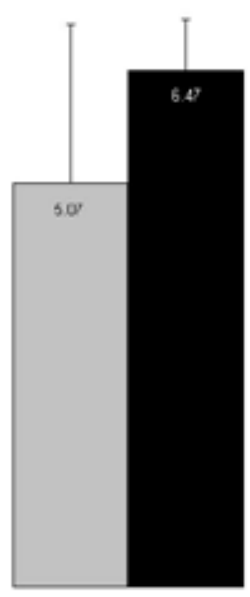

$p=0.023$

aManual

- Accactive Stinulater

Fig. 5. The mean (+1 SD) satisfaction rating of manual versus automatic adjustment during the standing to supine and supine to standing transitions is shown. Satisfaction ratings are shown on the 7-point Likert rating with 0 being very dissatisfied and 7 being very satisfied. The mean improvement in the satisfaction rating for automatic adjustment over manual adjustment for the standing to supine transition was 1.86 (SD 1.86) ( $P=0.01$, paired $t$-test). For the supine to standing transition, the mean improvement in the satisfaction rating was $1.40(S D 2.13)(P=0.023)$, paired $t$-test 


\section{Stimulation Amplitudes by Position}

Participants were asked about their preferred stimulation amplitudes in 7 positions (stand, sit, supine, on right side, on left side, prone, and recline at 45 degrees) and during a treadmill walk. Patient-preferred stimulation amplitudes represented as a percentage of the standing position amplitude are shown in Fig. 6A. On average, the supine position had the lowest amplitude relative to standing of $79.1 \%$. It should be noted that there was considerable inter-patient variability in the preferred mean stimulation amplitude by position. The relative percentages of preferred amplitudes for different positions compared to the standing position were statistically significant (Friedman's test, $\mathrm{P}<0.001$ ).

Individual variation in preferred amplitudes relative to standing by position for two participants labeled 101 and 213 is shown in Fig. 7A. The results indicated that these participants had definite preferential amplitudes in different positions. The lowest patient-preferred amplitude occurred in the supine position.

\section{Stimulation Impedance}

Stimulation impedances measured at patient-preferred amplitudes are presented as a percentage of the standing position impedance (Fig. 6B). The differences in impedance relative percentages were minor (Friedman's test, $P=0.373$ ), indicating that therapy (group) impedance is not statistically significant from position to position. The mean impedance variation (inter-quartile range $=4.5 \%$ ) across positions was small relative to the mean amplitude variation (inter-quartile range $=22.1 \%$ ).

The individual impedance profiles by position for patients 101 and 213 are shown in Fig. 7B. Participant 101 had minor variations in impedance by position, typical of participants in this study, while participant 213 had the greatest variation in impedance by position observed in the study.

\section{Adverse Events}

A total of 6 adverse events were reported during the study. There were no deaths or unanticipated adverse device effects, and none of these adverse events were considered to be related to study procedures or research devices used in the study. Three of the events were consequent to the implanted SCS lead impedance being out of range: one case was resolved with sequela; one without sequela; and one was not resolved. One of the additional 3 events was due to a depleted neurostimulator battery which was recharged, thereby resolving the event without sequela. The remaining two events involved worsening or exacerbation of a preexisting condition. One patient had congestive heart failure and was hospitalized. The situation was resolved without sequela. One patient had a pre-existing musculoskeletal and connective tissue disorder and developed

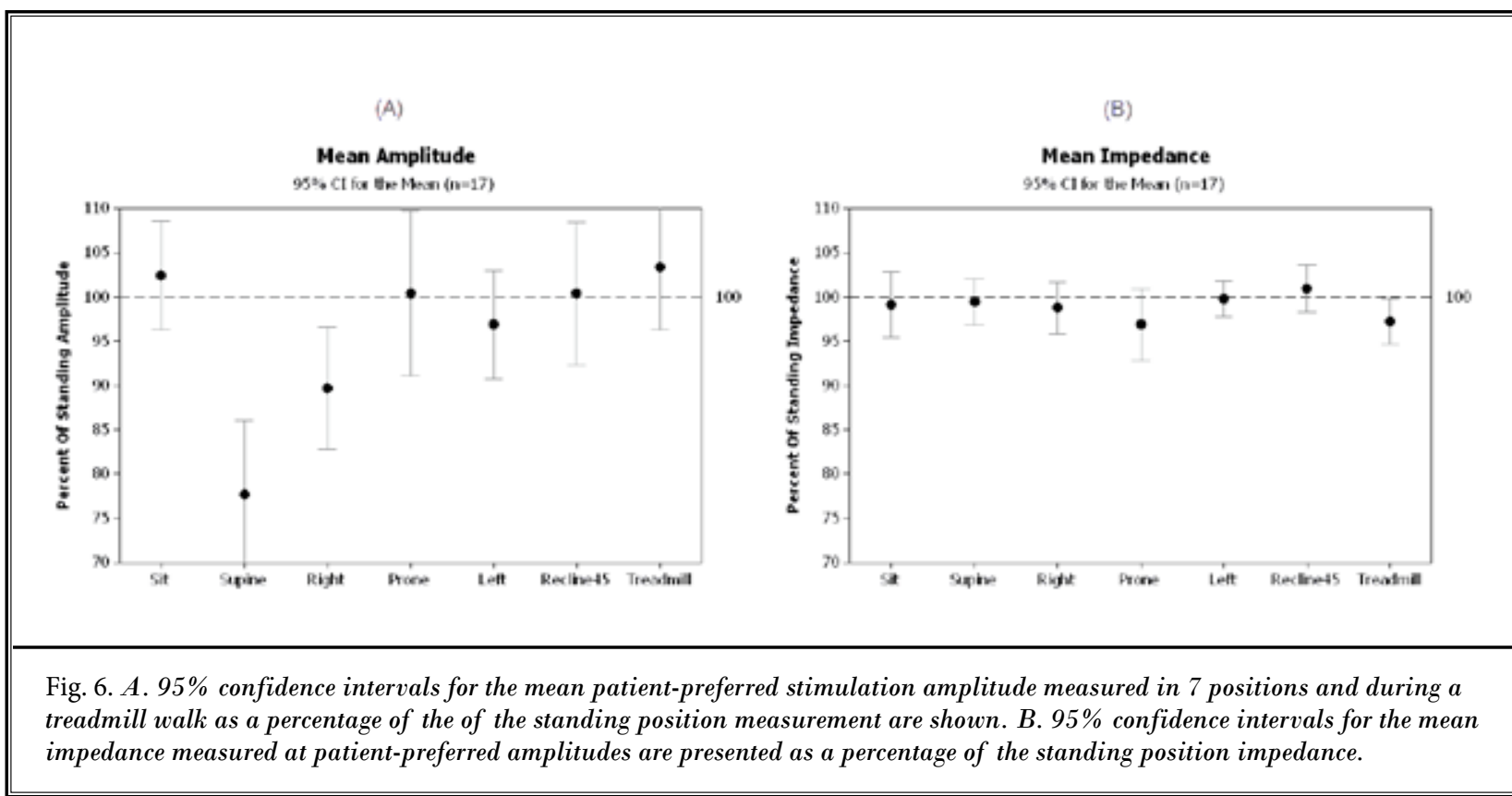




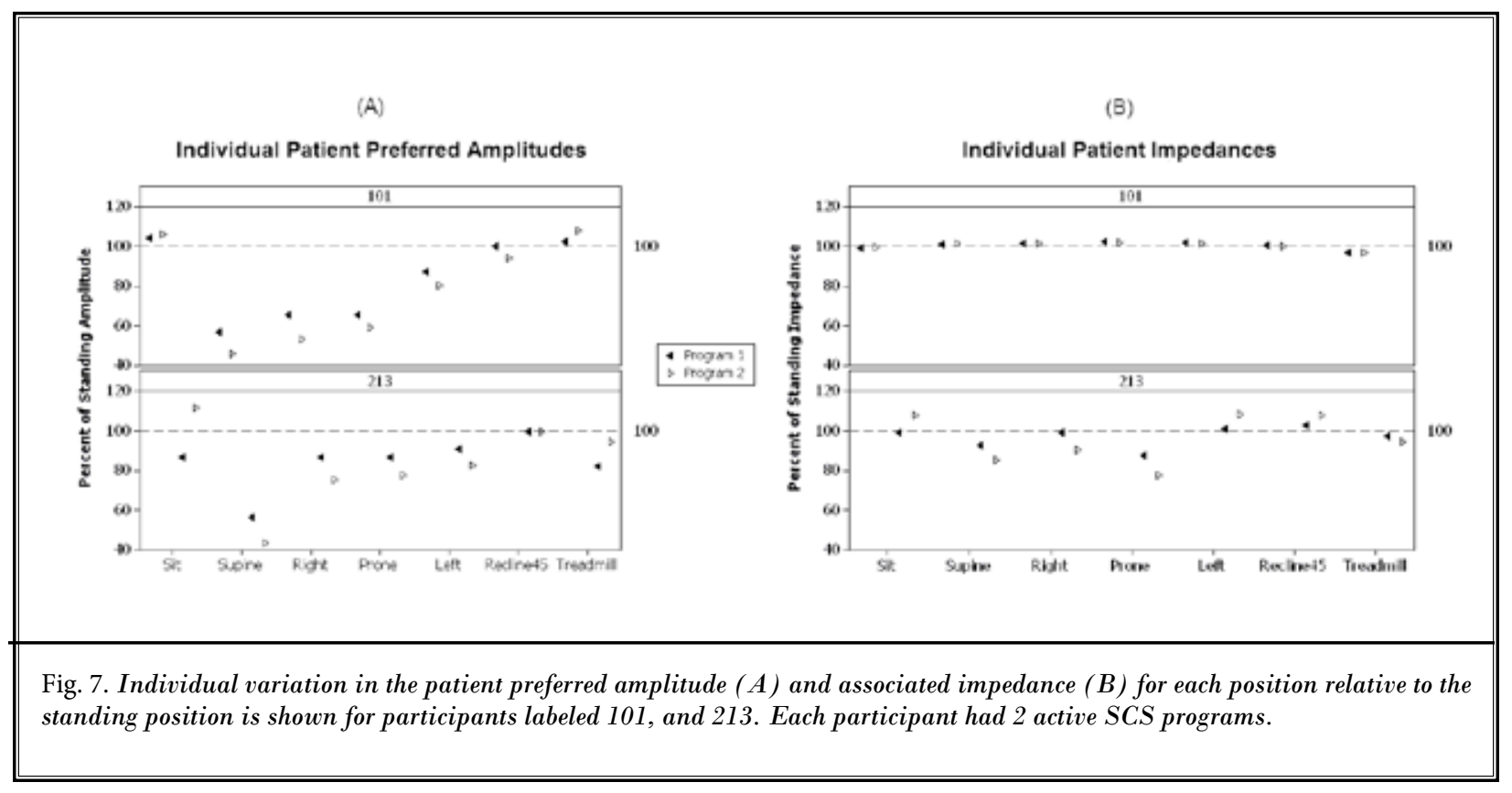

back spasms during the in-clinic phase of the study. The situation was resolved without sequela, and the patient was discontinued from the study.

\section{Discussion}

This prospective, multicenter study confirms the findings of previous studies that the stimulation required to achieve optimal paresthesia coverage and intensity varies according to the patient's position and that the supine position requires significantly less stimulation intensity when compared to the sitting or standing positions $(13,15)$. It also confirms the initial findings of Abejon and Feler that statistically significant differences have not been found in impedance with respect to position (11).

That postural changes impact the perception of paresthesia and play a role in the overall success of SCS in delivering optimal therapy has been observed in the clinical setting for many years. When patients change positions, they might receive stimulation levels perceived as shocking or jolting or alternatively as insufficient to cover their pain. As patient programmers with advanced capabilities have become more widely available, patients have had the option to manually adjust stimulation amplitude to a level that better suits the particular activity desired. However, despite efforts to develop user-friendly, menu-driven programmers, a certain percentage of patients do not have the facility or interest to use a patient programmer. Others, while initially using a programmer, use it less over time. The results of this study, as well as clinical experience, underscore the continuing unmet need for a more convenient and universally applicable method of adjusting stimulation to compensate for the many postural variations and transitions involved in activities of daily living.

This need has been studied theoretically and in humans (9). Anatomical studies using various imaging methods, including CT and MRI, have also been conducted to improve understanding of mechanisms $(8,15,17)$. This study represents a continuation of the work initiated by Cameron et al (12) and Olin et al (13) in characterizing the effects of postural changes on stimulation parameters.

Using MRI assessment, Holsheimer (15) found that the distance between stimulating electrodes and spinal cord dorsal columns, varied according to the spinal level and position of the patient. In the supine position, the dorsomedial CSF layer was thinnest at the midcervical level and thickest at the midthoracic level. At vertebral levels T-11 and T-12, the thickness increased when the patient turned from the supine to the prone position. At T-12, for example, the thickness increase associated with positional change was approximately $3.4 \mathrm{~mm}$ (15). Individual anatomic variations occurred in the CSF layer up to a factor of 2 . 
Accordingly, paresthesia perception will similarly vary depending on the vertebral level and patient position with increased dorsal CSF thickness raising the paresthesia threshold and a thinner layer lowering the threshold (18). These observations help explain why patients report uncomfortable stimulation levels when moving from the standing or sitting positions to lying positions. Furthermore, the CSF is a highly conductive medium and does not contribute significantly to the impedance from the stimulating electrode to spinal cord dorsal columns. This study confirms these clinical observations as demonstrated by patient-reported and measured preferred amplitudes and measured impedances.

A number of methods have been proposed previously to compensate for the postural effects in neurostimulation therapy including the application of constant current neurostimulation. The thinking has been that, in concordance with Ohm's Law, current delivered with a constant current neurostimulator automatically varies to compensate for changes in impedance resulting from postural changes. However, as Abejon and Feler (11) first demonstrated and our study confirms, impedance and position changes are not correlated. Therefore, an alternate technology is required to adjust neurostimulation automatically based on positional movement.

In this study a triaxial accelerometer was used to detect position changes and activity. The resulting data was used to automatically adjust neurostimulation amplitude to accommodate position changes and activity. The study demonstrated that patients have a statistically significant preference for automatic adjustment over manual adjustment. It also demonstrates the feasibility of using an implantable neurostimulation device that incorporates an accelerometer-based method of automatically adjusting stimulation based on position changes and activity. Such a medical device must be able to accommodate the large variation in preferred stimulation amplitude for a particular position observed in the study. A neurostimulator with capabilities to automatically adjust stimulation amplitude in response to changes in position and movement presents the possibility of improving neurostimulation therapy and patient quality of life and would be part of a general trend of increased automaticity in implantable medical devices. Furthermore, automated adjustment, position change, and activity data capture may change the nature of follow-up in SCS patients. Provision of usage data can be used in patient education as well as to track therapeutic progress and functional improvement.

\section{Limitations}

Testing was conducted in-clinic rather than in a free-living environment. In this open-label study, patients were aware they were receiving automatic adjustment of stimulation therapy, which created the possibility of unintentional bias. Although the patient sample was reflective of the population of patients receiving SCS therapy for chronic low back and leg pain and was satisfactory for feasibility purposes, its small size limits generalization to the overall population of chronic pain patients receiving SCS therapy.

\section{Conclusion}

This study demonstrated that patients using an implantable neurostimulation system were more satisfied with automatic versus manual adjustment of stimulation amplitude in response to positional changes and activity in an in-clinic setting. The preference for automatic adjustment included the standing to supine and supine to standing transitions. Additionally, the study demonstrated that patients have distinct position-dependent preferred stimulation amplitudes, whereas therapy impedance did not vary significantly relative to position. The study demonstrated both the value and the feasibility of implementing an accelerometer-based algorithm to automatically adjust neurostimulation parameters based on position and activity changes in an implantable neurostimulator.

\section{Disclosures}

Author Contributions: All authors contributed to the study design and execution and had full access to the study data. Study data was analyzed by Medtronic Neuromodulation. All authors provided critical review and final approval of the manuscript.

Conflict of Interest: The authors have no conflict of interest concerning the work under consideration for publication. The authors received no external funding for manuscript preparation. Drs. Schade and Schultz report serving as consultants for Medtronic, Inc., the study sponsor, for unrelated work. Ms. lyer managed the study for the sponsor, and Mr. Panken contributed to the design of the automatic adjustment algorithms under test and the study instrumentation.

Funding/Support: The study was funded and managed by Medtronic Neuromodulation.

The authors would like to thank Harriet Guthertz, an independent medical writer, who assisted in the preparation of this manuscript and Ye Tan, senior statistician, Medtronic Neuromodulation, who analyzed the study data. 


\section{References}

1. Manchikanti L, Boswell MV, Singh V, Benyamin RM, Fellows B, Abdi S, Buenaventura RM, Conn A, Datta S, Derby R, Falco FJE, Erhart S, Diwan S, Hayek SM, Helm S, Parr AT, Schultz DM, Smith HS, Wolfer LR, Hirsch JA. Comprehensive evidence-based guidelines for interventional techniques in the management of chronic spinal pain. Pain Physician 2009; 12:699-802.

2. Frey ME, Manchikanti L, Benyamin RM, Schultz DM, Smith HS, Cohen SP. Spinal cord stimulation for patients with failed back surgery syndrome: A systematic review. Pain Physician 2009; 12:379397.

3. Manchikanti L, Boswell MV, Datta S, Fellows B, Abdi S, Singh V, Benyamin RM, Falco FJE, Helm S, Hayek S, Smith HS. Comprehensive review of therapeutic interventions in managing chronic spinal pain. Pain Physician 2009; 12:E123E198.

4. Manchikanti L, Datta S, Gupta S, Munglani R, Bryce DA, Ward SP, Benyamin RM, Sharma ML, Helm II S, Fellows B, Hirsch JA. A critical review of the American Pain Society clinical practice guidelines for interventional techniques: Part 2. Therapeutic interventions. Pain Physician 2010; 13:E215-E264.

5. Kumar K, Taylor RS, Jacques L, Eldabe S, Meglio M, Molet J, Thomson S, O'Callaghan J, Eisenberg E, Milbouw G, Buchser E, Fortini G, Richardson J, North RB. The effects of spinal cord stimulation in neuropathic pain are sustained: a 24-month follow-up of the prospective randomized controlled multicenter trial of the effectiveness of spinal cord stimulation. Neurosurgery 2008; 63:762-770.

6. Kumar K, Taylor RS, Jacques L, Eldabe S, Meglio M, Molet J, Thomson S, O'Callaghan J, Eisenberg E, Milbouw G, Buchser E, Fortini G, Richardson J, North RB. Spinal cord stimulation versus conventional medical management for neuropathic pain: A multicentre randomised controlled trial in patients with failed back surgery syndrome. Pain 2007; 132:179-188.

7. North RB, Kidd DH, Farrokhi F, Piantadosi SA. Spinal cord stimulation versus repeated lubrosacral spine surgery for chronic pain: a randomized controlled trial. Neurosurgery 2005; 56:98-107.

8. He J, Barolat G, Holsheimer J, Struijk. Perception threshold and electrode position for spinal cord stimulation. Pain 1994; 59:55-63.

9. Barolat $\mathrm{G}$, Zeme S, Ketcik B. Multifactorial analysis of epidural spinal cord stimulation. Sterotact Funct Neurosurg 1991; 56-77-103.

10. Falowski S, Celii A, Sharan A. Spinal cord stimulation: an update. Neurotherapeutics 2008; 5:86-99.

11. Abejon D, Feler CA. Is impedance a parameter to be taken into account in spi- nal cord stimulation? Pain Physician 2007; 10:533-540.

12. Cameron T, Alo KM. Effects of posture on stimulation parameters in spinal cord stimulation. Neuromodulation 1998; 1:177-183.

13. Olin JC, Kidd DH, North RB. Postural changes in spinal cord stimulation perceptual thresholds. Neuromodulation 1998; 1:171-175.

14. Dijkstra EA, Holsheimer J, Olthuis W, Bergveld P. Ultrasonic distance detection for a closed-loop spinal cord stimulation system. Proc 1gth Ann Int Conf IEEE Eng in Med \& Biol Soc 1997; 5:19541957 .

15. Holsheimer J, den Boer JA, Rozeboom. $A R$ assessment of the normal position of the spinal cord in the spinal canal. Am J Neuroradiol 1994; 15:951-959.

16. Thomson S, Jacques L. Demographic characteristics of patients with severe neuropathic pain secondary to failed back surgery syndrome. Pain Pract 2009; 9:206-215.

17. Holsheimer J, Barolat G, Struijk J, He J. Significance of the spinal cord position in spinal cord stimulation. Acta Neurochir Wien Suppl 1995; 64:119-124.

18. Holsheimer J, Khan YN, Raza SS, Khan EA. Effects of electrode position on perception threshold and paresthesia coverage in spinal cord stimulation. Neuromodulation 2007; 10:34-41. 
